Europhys. Lett., 27 (3), pp. 175-180 (1994)

\title{
Non-Trivial Algebraic Decay in a Soluble Model of Coarsening.
}

\author{
A. J. BRaY $\left({ }^{*}\right)$, B. DeRRIDA $(* *)\left({ }^{* *}\right)$ and C. GodRÈche $\left({ }^{*}{ }^{*}\right)$ \\ Isaac Newton Institute for Mathematical Sciences \\ 20 Clarkson Road, Cambridge CB3 OEH, UK
}

(received 5 April 1994; accepted in final form 27 May 1994)

PACS. 02.50 - Probability theory, stochastic processes, and statistics.

PACS. 05.20 - Statistical mechanics.

\begin{abstract}
A non-trivial exponent $\beta$ characterising non-equilibrium coarsening processes is calculated in a soluble model. For a spin model, the exponent describes how the fraction $p_{0}$ of spins which have never flipped (or, equivalently, the fraction of space which has never been traversed by a domain wall) depends on the characteristic domain scale $L: p_{0} \sim L^{g-1}$. For the one-dimensional time-dependent Ginzburg-Landau equation at zero temperature we show that the critical exponent $\beta$ is the zero of a transcendental equation, and find $\beta=0.82492412 \ldots$.
\end{abstract}

Coarsening phenomena are rather common in physics. A typical example is the nonequilibrium evolution of the ordered domains that form when a system is thermally quenched from a homogeneous phase into a two-phase region [1]. Other examples include grain growth [2], soap froths [3], and breath figures [4].

A common feature of these phenomena is the scale-invariant morphology that develops at late times: the structure at different times is statistically similar apart from an overall change of scale, i.e. the system is described by a single, time-dependent length scale $L(t)$.

To fix our ideas, consider one of the simplest such systems-the $d=1$ Ising model, with Glauber dynamics, evolved from a random initial condition at temperature $T=0$. The behaviour of this system is well understood. The domain walls behave as independent random walkers. When two domain walls meet they annihilate. The average domain size $\langle l\rangle$ grows as $t^{1 / 2}$. The equal-time [5,6] and two-time [5] spin-spin correlation functions can be exactly calculated.

(*) Permanent address: Department of Theoretical Physics, The University, Manchester M13 9PL, UK.

(**) Permanent address: Laboratoire de Physique Statistique, Ecole Normale Supérieure, 24 Rue Lhomond, 75231 Paris Cedex 05, France.

(***) Permanent address: Service de Physique Théorique, CE Saclay, F-91191 Gif-sur-Yvette, France.

$\left({ }^{*}{ }^{*}\right)$ Permanent address: Service de Physique de l'Etat Condensé, CE Saclay, F-91191 Gif-sur-Yvette, France. 
Consider, however, the following question. How does the fraction $p_{0}$ of spins that have never flipped depend on time? Surprisingly, this seems to be a non-trivial question. Numerical simulations [7] indicate that $p_{0} \sim t^{-0.37}$, or, in terms of the mean domain size, $p_{0} \sim\langle l\rangle^{-0.74}$.

In this letter we address this question within the context of a simpler, deterministic model, namely the time-dependent Ginzburg-Landau equation in spatial dimension $d=1$, with no thermal noise (corresponding to $T=0$ ). The late-time dynamics of this model have been discussed by Nagai and Kawasaki [8], who showed that the distribution of domain sizes approaches a fixed-point distribution at late times. The equation of motion for the order-parameter field is $\partial_{t} \phi=\partial_{x}^{2} \phi-d V / d \phi$, where $V(\phi)$ is a symmetric double-well potential with minima at $\phi= \pm 1$, corresponding to the «up» and «down» phases of the Ising model. We consider an initial condition with a finite density of domain walls separating regions where $\phi$ is close to one of its equilibrium values. We take the initial distribution of domain wall sizes (or «intervals») $l$ to have a finite mean $\langle l\rangle$ much greater than the intrinsic width $\xi$ $\left(=\left[V^{\prime \prime}(1)\right]^{-1 / 2}\right)$ of the walls. Then the dynamics is very simple. Since the walls interact only through the exponential tails of the wall profile function, the closest pair of walls move together and annihilate, while the other walls hardly move at all. Thus the system coarsens by successively eliminating the boundaries of the smallest domain. As the wall density decreases, the domain size distribution $P(l)$ approaches the scaling form $P(l)=\langle l\rangle^{-1} f(l /\langle l\rangle)$. The scaling function $f(x)$ can be exactly calculated [8].

Introducing a terminology motivated by the study of breath figures [9], we shall call those parts of the line that have been traversed at least once by a domain wall «wet», and those parts not yet traversed "dry" (i.e. domain walls "wet» the line as they move). Each time a domain is eliminated, therefore, that part of the line which it occupied becomes wet. The density of dry region scales as $\langle l\rangle^{\beta-1}$ : this defines the exponent $\beta$. The dry part per domain increases as $\langle l\rangle^{\beta}$.

This procedure can be readily generalized to the $q$-state Potts model. The domains form a random sequence constructed from the $q$ available states (with no two consecutive states the same). Again, the smallest domain is identified. If the domains either side have the same state, the domain walls move together and annihilate. If they have different states, the two walls merge to form a new wall at the midpoint. In both cases, that part of the line previously occupied by the smallest domain becomes wet.

These models are easily simulated, and results for various values of $q$ are presented in fig. 1, in the form of a double-logarithmic plot of the dry part per unit length against the mean interval length. The asymptotic slope of the data therefore gives $\beta-1$. From the figure it is clear that $\beta$ decreases with increasing $q$, and becomes zero for $q=\infty$. This qualitative trend is easily understood. For the Ising case $(q=2)$, three intervals combine to form one larger interval at each step. The dry part of the new interval is the sum of the dry parts of the largest two of the intervals from which the new interval was formed. Therefore, the dry part per interval increases with time, and $\beta>0$. For $q=\infty$, three intervals combine to form two new intervals at each step. Each of these new intervals has the same dry part as the larger of the intervals from which it was formed: there is no tendency of the dry part per interval to increase, and $\beta=0$. For general $q$, behaviour intermediate between these two limits is expected, and observed.

For general $q$, it is not possible to calculate the asymptotic distribution of interval sizes, because these sizes become correlated, although approximate calculations neglecting these correlations are possible and have been carried out for $q=\infty$ (using the "cut in two" model of ref. [4]). For Ising systems $(q=2)$, however, initially uncorrelated interval sizes remain uncorrelated. If we choose $N$ intervals $I_{1}, \ldots, I_{N}$, the number of inequivalent arrangements of these intervals on a circle is $(N-1)$ !. The algorithm described above can be used to 


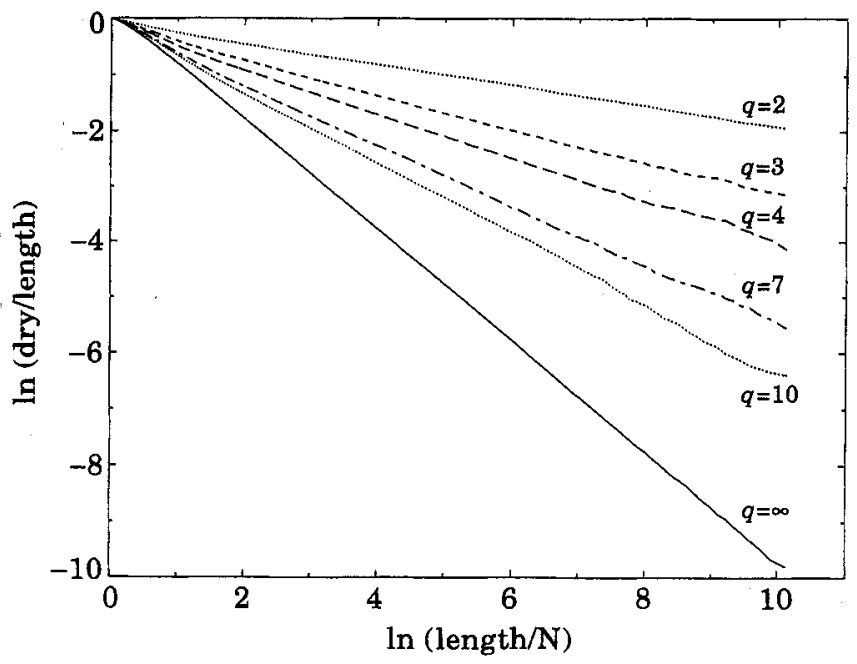

Fig. 1. - Log-log plot of the dry part per unit length against the mean interval size, for various values of $q$, the number of Potts states. In each case the initial state consisted of $10^{5}$ intervals, with lengths uniformly distributed between 0.5 and 1.5 . The asymptotic slope of each line gives $1-\beta$. The estimates for $\beta$ are (top to bottom) $0.82,0.67,0.58,0.44,0.36$, and zero, for $q=2,3,4,7,10$ and $\infty$, respectively.

deterministically coarsen each of these systems to a single interval. Now consider an alternative procedure in which the same $N$ intervals are initially placed in a bag. The new procedure consists of taking the smallest interval from the bag, combining it with two intervals chosen at random, replacing the new interval in the bag, and then iterating the procedure until a single interval remains (if there is an even number of intervals, only two intervals are combined at the final step). The $(N-1)$ ! possible histories generated by this alternative procedure are in one-to-one correspondence with the deterministic histories produced by the original algorithm applied to the inequivalent arrangements of the intervals on a circle. This proves that no correlations develop. As a result, both the limiting distribution of interval sizes and the exponent $\beta$ can be calculated exactly for $q=2$ by using the bag (which is in fact a mean-field) model.

The calculation of $\beta$ in the Ising case proceeds as follows. We start with random intervals on the line. Each interval $I$ is characterised by its length $l(I)$ and by the length of its dry part $d(I)$. At each time step, the smallest interval $I_{\min }$ is removed. So three intervals (the smallest interval $I_{\min }$ and its two neighbours $I_{1}$ and $I_{2}$ ) are replaced by a single interval $I$. The total length and the dry parts of $I$ are given by

$$
l(I)=l\left(I_{1}\right)+l\left(I_{\min }\right)+l\left(I_{2}\right), \quad d(I)=d\left(I_{1}\right)+d\left(I_{2}\right) .
$$

The calculation is made tractable by the fact that the lengths of the intervals remain uncorrelated: one can choose the intervals $I_{1}$ and $I_{2}$ randomly, instead of choosing the neighbours of the smallest interval. Note that the number $\nu(I)$ of dry regions in the interval $I$ satisfies the same recursion equation as $d(I)$.

For simplicity of presentation, we will assume that the lengths of the intervals take only integer values and that the minimal length in the system is $i_{0}$ (the calculation can also be done on the continuum - the final result is the same). We assume that there is a very large number $N$ of intervals, that the number of intervals of length $i$ is $n_{i}$ and that the average length of the 
dry part of the intervals of length $i$ is $d_{i}$. By denoting with a prime the values of these quantities after all the $n_{i_{0}}$ intervals of length $i_{0}$ have been eliminated, so that the minimal length has become $i_{0}+1$, the time evolution is given by

$$
\left\{\begin{array}{l}
N^{\prime}=N-2 n_{i_{0}}, \\
n_{i}^{\prime}=n_{i}\left(1-\frac{2 n_{i_{0}}}{N}\right)+n_{i_{0}} \sum_{j=i_{0}}^{i-2 i_{0}} \frac{n_{j}}{N} \frac{n_{i-j-i_{0}}}{N}, \\
n_{i}^{\prime} d_{i}^{\prime}=n_{i} d_{i}\left(1-\frac{2 n_{i_{0}}}{N}\right)+n_{i_{0}} \sum_{j=i_{0}}^{i-2 i_{0}} \frac{n_{j}}{N} \frac{n_{i-j-i_{0}}}{N}\left(d_{j}+d_{i-j-i_{0}}\right) .
\end{array}\right.
$$

This is only valid under the condition that $n_{i_{0}} \ll N$, which is indeed valid when $i_{0}$ becomes large.

We assume that after many iterations, i.e. when $i_{0}$ becomes large, a scaling regime is reached, where

$$
n_{i}=\frac{N}{i_{0}} f\left(\frac{i}{i_{0}}\right), \quad n_{i} d_{i}=N i_{0}^{\beta-1} g\left(\frac{i}{i_{0}}\right) .
$$

Since $i_{0}$ is large, one can treat $x=i / i_{0}$ as a continuous variable. Then we can write

$$
\left\{\begin{array}{l}
n_{i}^{\prime}=\frac{N^{\prime}}{i_{0}+1} f\left(\frac{i}{i_{0}+1}\right)=\frac{N}{i_{0}}\left[f(x)-\frac{2}{i_{0}} f(1) f(x)-\frac{1}{i_{0}} f(x)-\frac{1}{i_{0}} x f^{\prime}(x)\right], \\
n_{i}^{\prime} d_{i}^{\prime}=N^{\prime}\left(i_{0}+1\right)^{\beta-1} g\left(\frac{i}{i_{0}+1}\right)=N i_{0}^{3-1}\left[g(x)-\frac{2}{i_{0}} f(1) g(x)+\frac{\beta-1}{i_{0}} g(x)-\frac{1}{i_{0}} x g^{\prime}(x)\right],
\end{array}\right.
$$

where higher-order terms in $1 / i_{0}$ are negligible in the scaling limit. Inserting these expressions in the time evolution equations (2), and using the fact that the functions $f$ and $g$ become independent of $i_{0}$ for large $i_{0}$, gives

$$
\left\{\begin{array}{l}
f(x)+x f^{\prime}(x)+\theta(x-3) f(1) \int_{1}^{x-2} \mathrm{~d} y f(y) f(x-y-1)=0 \\
(1-\beta) g(x)+x g^{\prime}(x)+2 \theta(x-3) f(1) \int_{1}^{x-2} \mathrm{~d} y g(y) f(x-y-1)=0
\end{array}\right.
$$

These equations are easily solved by introducing the Laplace transforms

$$
\phi(p)=\int_{1}^{\infty} \exp [-p x] f(x) \mathrm{d} x, \quad \psi(p)=\int_{1}^{\infty} \exp [-p x] g(x) \mathrm{d} x,
$$

which satisfy the equations

$$
\left\{\begin{array}{l}
p \phi^{\prime}(p)=-f(1) \exp [-p]\left[1-\phi^{2}(p)\right] \\
p \psi^{\prime}(p)+\beta \psi(p)=-\exp [-p][g(1)-2 f(1) \phi(p) \psi(p)] .
\end{array}\right.
$$

It is convenient to introduce the function $h(p)$ defined by

$$
h(p)=2 f(1) \int_{p}^{\infty} \frac{\mathrm{d} t}{t} \exp [-t]
$$


Then the solutions of eqs. (7) can be written in the form

$$
\begin{gathered}
\phi(p)=\operatorname{tgh}[h(p) / 2], \\
\psi(p)=g(1) \int_{p}^{\infty} \frac{\cosh ^{2}[h(q) / 2]}{\cosh ^{2}[h(p) / 2]} \frac{q^{\beta-1}}{p^{\beta}} \exp [-q] \mathrm{d} q .
\end{gathered}
$$

When integrating (7), the particular choices for the constants of integration, implied by the forms (9) and (10), were fixed by the requirement that both $\phi$ and $\psi$ be positive for all $p$ (as is clear from their definitions (6)).

So far, the constants $f(1)$ and $\beta$ are arbitrary. Both are fixed, however, by physical considerations. The following expansion will prove useful:

$$
\int_{p}^{\infty} \frac{\mathrm{d} q}{q} \exp [-q]=-\ln p-\gamma-\sum_{n=1}^{\infty} \frac{(-p)^{n}}{n n !}
$$

where $\gamma$ is Euler's constant, given by $\gamma=-\int_{0}^{\infty} \mathrm{d} t \ln t \exp [-t]=0.577215 \ldots$. Using this with eqs. (8) and (9) gives the small-p expansion

$$
\phi(p)=1-2 \exp [2 f(1) \gamma] p^{2 f(1)}[1+O(p)] .
$$

From the definition (6) of $\phi(p)$, however, we have the expansion $\phi(p)=1-\langle x\rangle p+\ldots$, provided the first moment of the distribution $f(x)$ exists. Comparing the two expansions fixes $f(1)=1 / 2$, and also gives the first moment of the scaling function $f$ as $\langle x\rangle=2 \exp [\gamma]=$ $=3.56214 \ldots$, this being the ratio of the mean domain length to the minimum length. This latter result is in agreement with ref. [8], as is eq. (9) for the Laplace transform of the distribution of interval sizes. (Since the first moment cannot vanish, we have quite generally $f(1) \leqslant 1 / 2$. The cases $f(1)<1 / 2$ would correspond to initial conditions with infinite first moments [4]: we will not consider these cases further.)

The exponent $\beta$ can be similarly determined. If one defines $r(p)$ by

$$
r(p)=h(p)+\ln p=-\gamma-\sum_{n=1}^{\infty} \frac{(-p)^{n}}{n n !},
$$

one can rewrite (10) as

$$
\psi(p)=g(1) \int_{p}^{\infty} \frac{\exp [r(q)]+2 q+q^{2} \exp [-r(q)]}{\exp [r(p)]+2 p+p^{2} \exp [-r(p)]} \frac{q^{\beta-2}}{p^{\beta-1}} \exp [-q] \mathrm{d} q .
$$

Note that $r(p)$ can be expanded in powers of $p$ using (13), so eq. (14) is a convenient starting point for investigating the small-p behaviour of $\psi(p)$. A straightforward calculation gives

$$
\psi(p)=\frac{g(1)}{1-\beta}\left(1+B(\beta) p^{1-\beta}+O(p)\right)
$$

where

$$
\begin{aligned}
B(\beta)=\exp [\gamma] \int_{0}^{\infty} \mathrm{d} q q^{\beta-2} \exp [-q] . & \\
& \cdot\left[(1-q-\exp [-q]) \exp [r(q)]+2(1-\beta) q+(1-\beta) q^{2} \exp [-r(q)]\right] .
\end{aligned}
$$

In the spirit of our earlier determination of $f(1)$, we compare the expansion (15) with a 
direct expansion of eq. (6), namely $\psi(p)=\int_{1}^{\infty} \mathrm{d} x g(x)\left(1-p x+O\left(p^{2}\right)\right)$. If the function $g(x)$ is to have a finite first moment, then we must have $B(\beta)=0$ in (16). This gives $\beta=\beta_{0}=$ $=0.82492412 \ldots$. It is remarkable that the only limitation on the accuracy of $\beta$ is the numerical effort expended in evaluating the integral (16). It is easy to show that all higher terms in the expansion (15) involve only integer powers of $p$. The exact result for $\beta$ is in good agreement with the estimate $\beta \simeq 0.82$ from the simulation (fig. 1). This non-trivial value of a critical exponent is reminiscent of what has been seen in various irreversible systems [10].

Note that, since $\psi(p)$ decreases with $p$, one must have $B(\beta) \leqslant 0$ in (16). A study of (16) shows that this implies $\beta \geqslant \beta_{0}$. The cases $B<0$, however, correspond to a $g(x)$ with a power law tail $x^{-(2-\beta)}$. It can be shown that when, as here, such a tail is absent from the initial condition it cannot be generated. This is why we selected the value $\beta=\beta_{0}$, corresponding to $B(\beta)=0$.

A possible extension of the present work is to higher dimensions, where the motion of the domain walls is still deterministic, but is curvature driven. This seems to be a non-trivial problem deserving further study. Simulation results for the corresponding stochastic Ising model (i.e. Glauber dynamics at $T=0$ ) are reported in [7]. In one dimension, the deterministic model studied here gives a dry part which scales as $\langle l\rangle^{-0.175}$, whereas the $1 d$ Ising model with stochastic dynamics gives $\langle l\rangle^{-0.74}$. It would be interesting to see if this difference persists in higher dimensions.

A related area is that of breath figures. Recent work in this field [9] was indeed one of the driving forces behind the present paper. In these experiments, droplets grow and coalesce on a substrate. The part of the surface which has never been wetted by a droplet (the «dry» region) therefore decreases with time. Both in the experiments, and in simulations of simple models inspired by them, this decrease was found to be algebraic in time in the scaling region [9].

We thank the Isaac Newton Institute for Mathematical Sciences, where this work was carried out, for its hospitality, and D. Beysens, J. P. Bouchaud, M. Marcos and I. YEKUTIELI for useful discussions.

\section{REFERENCES}

[1] See, e.g., Gunton J. D., San Miguel M. and Sahni P. S., in Phase Transitions and Critical Phenomena, edited by C. Domb and J. L. Lebowitz, Vol. 8 (Academic Press, New York, N.Y.) 1983, p. 267; BRAY A. J., in Phase Transitions in Systems with Competing Energy Scales, edited by T. Riste and D. Sherrington (Kluwer Academic) 1993.

[2] Anderson M. P., Srolovitz D. J., Grest G. S. and Sahni P. S., Acta Metall., 32 (1984) 783.

[3] FlyvbJerg H. and Jeppesen C., Phys. Scr., T38 (1991) 49.

[4] Derrida B., Godrèche C. and Yenutieli I., Phys. Rev. A, 44 (1991) 6241; Europhys. Lett., 12 (1990) 385.

[5] Bray A. J., J. Phys. A, 23 (1990) L-67.

[6] Amar J. G. and Family F., Phys. Rev. A, 41 (1990) 3258.

[7] Derrida B., Bray A. J. and Godrìche C., to be published in J. Phys. A.

[8] Nagai T. and Kawasaki K., Physica A, 134 (1986) 483; Rutenberg A. D. and Bray A. J., submitted to Phys. Rev. E.

[9] Marcos M., Beysens D., Bouchaud J. P., Godrèche C. and Yekutieli I., in preparation.

[10] Tarjus G. and Viot P., Phys. Rev. Lett., 67 (1991) 1875; Sherwood J. P., J. Phys. A, 23 (1990) 2827; Leyvraz F. and Redner S., Phys. Rev. Lett., 66 (1991) 2168; Phys. Rev. A, 46 (1992) 3132; Cornell S., Droz M. and Chopard B., Physica A, 188 (1992) 322. 